\title{
Advances in Pulp Capping Materials: A Review
}

\author{
Dr. Seema D.Pathak ${ }^{1}$, Dr. Pradnya V.Bansode ${ }^{2}$, \\ Dr. M. B. Wavdhane ${ }^{3}$, Dr. Shirish Khedgikar ${ }^{4}$, \\ Dr. Priyanka P. Birage ${ }^{5}$ \\ ${ }^{1}$ Associate Professor, (Department Of Conservative Dentistry And Endodontics, Gdc \& Hospital, \\ Aurangabad/Muhs, India) \\ ${ }^{2}$ Professor And Head Of Department, (Department Of Conservative Dentistry And Endodontics, Gdc \& \\ Hospital, Aurangabad/Muhs, India). \\ ${ }^{3}$ Associate Professor, (Department Of Conservative Dentistry And Endodontics, Gdc \& Hospital, \\ Aurangabad/Muhs, India) \\ ${ }^{4}$ Lecturer, (Department Of Conservative Dentistry And Endodontics, Gdc \& Hospital, \\ Aurangabad/Muhs, India) \\ ${ }^{5}$ First Year Post-Graduate Student, (Department Of Conservative Dentistry And Endodontics, Gdc \& \\ Hospital, Aurangabad/Muhs, India).
}

\begin{abstract}
Vital pulp therapy has been known as one of the treatment options to preserve pulp after being exposed by trauma or caries. The procedure of pulp capping relies primarily on the ability of pulpal tissue to heal. A wide array of materials has been used for pulp capping. The aim of this article is to summarize and discuss various pulp capping materials.
\end{abstract}

Keywords : Bioactive cement, Biocompatible, Dentin bridge, Pulp capping, Pulp - dentin complex, Vital pulp therapy.

\section{Introduction}

The foundation of restorative dentistry rests on the principle that the maintenance of a healthy and functional pulp-dentine complex would result in the successful healing of the exposed pulp. Like other connective tissue, pulp tissue has the potential to heal [1]. A medicament is placed directly over the exposed pulp (direct pulp cap), or a cavity liner or sealer is placed over residual tooth structure in an attempt to maintain pulp vitality.Key responses of the dentin-pulp to injury include the deposition of tertiary dentin, which increases the distance between the injury and the pulp, and a reduction in dentin permeability by the formation of sclerotic dentin. Historically, the first pulp capping procedure was performed in 1756, by Phillip Pfaff, who packed a small piece of gold over an exposed vital pulp to promote healing [4]. Calcium hydroxide is one of the oldest and most widely used medicaments for stimulation of dentinal bridge formation subsequent to microscopic or gross pulpal exposure [3]. Now a days many materials are available for pulp capping procedure and this paper will review various pulp capping materials.

\section{The Pulp Capping Material Should Have The Following Ideal Properties Like}

- Stimulate reparative dentin formation

- Maintain pulpal vitality

- Bactericidal or bacteriostatic

- Adhere to restorative material

- Resist forces during restoration placement and during the life of restoration.

- Sterile

- Radiopaque

- Provide bacterial seal [5]

\section{Calcium hydroxide}

Calcium hydroxide was introduced to the dental profession in 1921 by Hermann and has been considered the 'gold standard' of direct pulp capping materials for several decades because of their property of stimulating sclerotic and reparative dentin formation and protecting the pulp against thermal stimuli and antibacterial action. Calcium hydroxide serves as protective barrier for pulpal tissues not only by blocking patent dentinal tubules but also by neutralizing the attack of inorganic acids and their leached products from certain cements and filling materials. Mitchell and Shankhwalkar suggested that calcium hydroxide has unique potential to induce mineralization even in tissues that have not been programmed to mineralize. [6] 
Sciaky and Pisanti in 1960 observed that calcium hydroxide does not become incorporated in the mineralized repaired tissues, which derives its mineral content solely from the dental pulp, presumably via the blood supply.Forman observed that calcium hydroxide is an initiator rather than substrate for repair. [6]

Some failures may result from faulty techniques when extrapulpal blood clots are left between the calcium hydroxide and the cut tissue. In these cases the hydroxyl ions would be trapped in the clot and the requisite induction of odontoblast differentiation does not occur. [6]

During healing 3 zones are formed:

-Zone of obliteration: The pulp tissue immediately in contact with the calcium hydroxide is usually completely deranged and distorted because of the caustic effect of the drug. This zone consists of debris, dentinal fragments, hemorrhage, blood clot, blood pigment, and particles of calcium hydroxide. Schoder and Granath explained that this zone of obliteration was due to the chemical injury due to a high concentration of hydroxyl ions and the high pressure of the medicament application.

-Zone of coagulation necrosis: The tissue together with its plasma proteins within the zone of obliteration takes the brunt of the calcium hydroxide chemical thrust. A weaker chemical effect reaches the subjacent, more apical tissues and results in a zone of coagulation necrosis and thrombosis, also called Schroder's layer of 'firm necrosis' and Stanley's 'mummified zone'. This zone of coagulation necrosis ranges from 0.3-0.7 mm thickness.

-Zone of demarcation: A line of demarcation develops between the deepest level of the zone of coagulation necrosis and the subjacent vital pulp tissue. Glass and Zander believed that this line resulted from the reaction of the calcium hydroxide with the tissue protein to form proteinate globules. The study conducted by R. Kemal Subayhas verified that exposed human dental pulp will heal under Dycal application with hard tissue bridging and with none or minimal inflammatory response. [7] However, the importance of calcified hard tissue barrier formation after capping has been challenged by other studies, which have shown multiple tunnel defects and cell inclusions in bridges following pulp capping with calcium hydroxide. This may lead to leakage and bacteria penetration into pulp tissue unlike the permanent seal produced by bonding agents. [8]

\section{Adhesive System}

Adhesive resins are almost equal to calcium hydroxide with regard to dentin bridge formation and wound healing. However, dentin bridge formation is initiated earlier in calcium hydroxide capped teeth. [9]

According to study done by Carlos Alberto De Souza Costa on first molars of rats; despite the inflammatory response caused by the experimental (Clearfil Liner Bond 2Vand Vitrebond) and the control materials (a calcium hydroxide/saline paste) at 7 days; pulpal healing associated with calcified barrier formation was observed at 60 days following the pulp therapy. Both resin-based materials promoted a large zone of cellrich fibrodentine matrix deposition on the pulp horn related to the pulp exposure site. [10]

In vivo studies have demonstrated that the application of an adhesive resin directly onto a site of pulp exposure, or to a thin layer of dentin $(<0.5 \mathrm{~mm})$, causes dilatation and congestion of blood vessels, inflammation, and pulp abscesses. Importantly, no dentin bridge can be seen in the majority of human teeth treated with direct pulp capping with adhesive resin. The lack of dentin bridging might render the pulp more susceptible to inflammation mediated by bacterial contamination if microleakage is observed at the resin-tooth interface. Complete polymerization of adhesive resins might be unachievable during direct pulp-capping procedures. Oxygen was shown to prevent complete polymerization of adhesive resin monomers, and hemorrhagic sites tend to have high oxygen tension. Humidity may also prevent complete polymerization of adhesive resin, and a site of pulp exposure tends to be humid due to the presence of blood/clot and exudates. Unpolymerized monomers can diffuse directly into the pulp at the exposure site, as well as diffuse through the dentinal tubules, and cause cytotoxic effects in pulp cells. [11]

\section{Calcium Phosphate Cement (Cpc)}

Calcium phosphate is another pulp capping agent which came into practice in the 1900's. Its advantage is it helps in forming dentin bridge without any tissue necrosis. It has good physical properties. Also the absence of pulp inflammation is seen compared to calcium hydroxide. Release of calcium ions is the key factor for a successful pulp capping because of action of calcium on differentiation, proliferation and mineralisation of pulp cells. But calcium phosphate is not commonly used till now. Because more clinical trials are necessary to evaluate the material.The results of study done by Chaung HM showed that calcium phosphate cement and pure calcium hydroxide materials produced similar responses with regard to their biocompatibility and induction of hard tissue barrier formation. A more mature, hard tissue barrier with a better degree of mineralization and formation of dentinal tubules was demonstrated after 24-week. Calcium phosphate cement appears superior to pure calcium hydroxide and may have potential for clinical application, although many issues remain to be further investigated. [13] 


\section{Hydroxyapatite}

The results of study done by R. Kemal Su'bay showed that hydroxyapatite does not induce hard tissue bridging at the exposure site in human dental pulp. Moderate infiltration of the inflammatory cells was seen generally in the teeth capped with hydroxyapatite. [7]

According to R. M. Frank a continuous, well-calcified bridge was observed and no inflammatory cells were present in the residual pulp. In some cases, denticles containing some synthetic hydroxyapatite crystals were found in the radicular pulp. The calcified bridge was made up by osteodentine and near the pulp, tubular dentine with a layer of predentine and odontoblasts were noted. Odontoblastic processes were located in deninal tubules and the intertubular dentine showed interwoven calcified collagen fibrils.[14]

\section{Lasers}

Lasers offer excellent characteristics in terms of hemostasis and decontamination for field preparation during direct pulp capping treatment; however, the sealing of exposed pulp with one of the dental materials, such as calcium hydroxide, mineral trioxide aggregates, and bonded composite resins, is still required after laser treatment. Due to their excellent results for hemostasis and decontamination, lasers have grown in popularity for direct pulp capping in the clinic. [15]

Laser-assisted pulp capping has considerable advantages compared to traditional methods

Basically they can be defined as:

1- Decontaminant effect;

2- Haemostatic and coagulant effect;

3- Reduced rise in pulp temperature;

4- Reduction of intracavitary pressure;

5- Dentinal melting;

6- Biostimulating effect.

The sterile field is provided by the bactericidal effect of the laser, an effect common to all lasers, which however takes place differently according to the wavelength. $\mathrm{CO}_{2}$ and lasers of the Erbium family are more superficial than Diode and Nd:YAG lasers, which penetrate more deeply and have a greater scatter capacity. This effect is especially important in the event of carious exposures where the indications for pulp capping are still present.

The haemostatic and coagulant capacities of lasers guarantee a dry operative area with no bleeding. This fine area of coagulation is created by a superficial necrosis, with an underneath area of reversible damage, this is the seat of migration of the inflammatory cells and the fibroblasts that lead to the constitution of the dentinal bridge. [16]

\section{Mineral Trioxide Aggregate (Mta)}

MTA basically consists of calcium, silica, and bismuth oxide. After hydration MTA releases calcium hydroxide that is the reason of the high $\mathrm{pH}$ value of the material. It produces more dentinal bridging with superior structural integrity than $\mathrm{Ca}(\mathrm{OH})_{2}$ in a shorter time span with significantly lesser inflammation. It has superior ability to resist for further penetration of bacteria than calcium hydroxide. Has significant antimicrobial property on some of the facultative bacteria.MTA is highly biocompatible with pulpal tissue and it is hydrophilic as it sets in presence of moisture. Set MTA has $\mathrm{pH}$ of 12.5 and may induce dentinogenesis. The presence of blood has little impact on degree of leakage of MTA.

In vitro and in vivo studies confirmed MTA's excellent sealing ability and biocompatibility. [17] According to M. Aeinehchi et al after six month follow up using MTA a $0.43 \mathrm{~mm}$-thick dentine bridge and a nearly regular odontoblastic layer were noted. No inflammation, necrosis or calcifications were registered. After Six-month follow up using calcium hydroxide, calcification and necrosis were seen underneath a bridge of maximum 0.15 mm thickness. Two types of MTA: White and Gray MTA The major difference between GMTA and WMTA are the presence of the higher amount of aluminum, magnesium, and iron in the former material. Because of potential discoloration effect of gray MTA, white MTA has been introduced into endodontic treatment.According to Ali Eskandarizadeh et al no significant difference was found between GMTA and WMTA in terms of calcified bridge thickness and pulp inflammatory response to the capping materials [17]

The powder of MTYA1-Ca is composed of

VII. Mtya1-Ca

$89.0 \%$ microfiller,

$10.0 \%$ calcium hydroxide and

$1.0 \%$ benzoyl peroxide and

-was mixed with liquid 
$67.5 \%$ triethyleneglycoldimethacrylate,

$30.0 \%$ glyceryl methacrylate,

$1.0 \%$ o-methacryloyl tyrosine amide,

$1.0 \%$ dimethylaminoethylmethacrylate,

and $0.5 \%$ camphorquinone

Niinuma A studied that the physical properties of MTYA1-Ca were significantly superior to those of Dycal. MTYA1-Ca maintained high levels of alkaline activity (pH 10.96-12.20) over the 168 hours' duration of the study. Cell viability by MTYA1-Ca was significantly higher than that of Dycal, A dentine bridge formed more slowly under MTYA1-Ca than under Dycal, but similar amounts had formed at 90 days. [19]

\section{Growth Factors}

Growth factors are peptide molecules that transmit signals between cells functioning as stimulators and/or inhibitors of growth as well as modulators of differentiation state amongst other roles.

The potential functional activities of the growth factors sequestrated within dentin matrix may be quite extensive. In addition to the induction of odontoblast- like cell differentiation, these molecules may signal cell proliferation and migration within the pulp.

A number of reports of the in vivo or in vitro placement of exogenous growth factors, particularly TGF (Transforming Growth Factor) and Bone Morphogenetic Proteins (BMPs), on exposed pulps have demonstrated the potential of these molecules to signal reparative dentinogenic events. Transdentinal or direct application of TGF-1 and BMP-7 to the odontoblasts of unexposed pulps in cultured tooth slices has also shown the ability of these growth factors to signal reactionary dentinogenesis.[20]

Pulps capped with BMP-7 demonstrated a hard tissue that was bone-like in appearance. [21]

Lovschall $\mathrm{H}$ did study on rat molars using recombinant human insulin-like growth factor I (rhIGFI)and they found after 28 days, complete dentin bridging and tubular dentin formation were observed.[22]

\section{Bonesialoprotein (BSP)}

Bone sialoprotein (BSP) is a major non-collagenous protein in mineralizing connective tissues such as dentin, cementum and calcified cartilage tissues. BSP is involved in regulating hydroxyapatite crystal formation in bones and teeth. According to study done by M. GoldbergBSP-implanted group, the osteogenic protein stimulated the formation of a homogeneous dentin-like deposit occupying most of the mesial part of the pulp. Apparently, BSP stimulates the differentiation of cells which secrete an organized extracellular matrix more efficiently than any other capping material used so far. Altogether, the results reported here support that bone sialoprotein displays novel bioactive properties and is capable of stimulating in 1 months' time the development of a thick reparative dentinal tissue in the pulp, occluding the perforation and filling the mesial third of the pulp chamber.[24]

\section{Biodentin}

Biodentin was developed by Septodont's Research Group as a new class of dentin material which could conciliate high mechanical properties, excellent biocompatibility and bioactive behavior. It was developed as a silicate based restorative material. Due to its good sealing ability with dentin, it is used as a dentin replacement material. Biodentin has its use both in coronal part of tooth and in roots. It also has shorter setting time thus eliminating the drawbacks of MTA. [25]

It has dentin-like mechanical properties, which may be considered a suitable material for clinical indications of dentin-pulp complex regeneration such as direct pulp capping. It has a positive effect on vital pulp cells and stimulates tertiary dentin formation.

The powder mainly contains tricalcium and dicalcium silicate $\left(3 \mathrm{CaO} \mathrm{SiO} \mathrm{S}_{2}\right.$ and $\left.2 \mathrm{CaO} \mathrm{SiO}_{2}\right)$, the principal component of Portland cement, as well as calcium carbonate $\left(\mathrm{CaCO}_{3}\right)$. Zirconium dioxide $\left(\mathrm{ZrO}_{2}\right)$ serves as contrast medium. The liquid consists of calcium chloride $\left(\mathrm{CaCl}_{2} \cdot \mathrm{H} 2 \mathrm{O}\right)$, which is used as a setting accelerator and water-reducing agent in aqueous solution with an admixture of polycarboxylate (a superplasticizing agent)

According to Alicja Nowicka Biodentine had a similar efficacy in the clinical setting and may be considered an interesting alternative to MTA in pulp-capping treatment during vital pulp therapy. [26]

\subsection{Heme-Oxygenase-1}

\section{Enzymes}

Heme oxygenase-1 (HO-1) activity is related to stem cell differentiation.

According to Sun-Ju Kim HO-1 induction by cobaltic protoporphyrin IX (CoPP) in human dental pulp cells (HDPCs) increased cell growth and mineralization. Pharmacologic HO-1 induction might represent a potent therapeutic approach for pulp capping and the regeneration of human dental pulp cells. [27] 


\subsection{Simvastatin}

According to Kyung-San Min Simvastatin enhanced the differentiation of human dental pulp cells by up-regulating mineralization nodules and odontogenic markers as well as angiogenic markers.[28]

\section{Propolis (Russian penicillin)}

Propolis is collected from trees and shrubs by honeybees. The main chemical classes present in propolis are flavonoids, phenolics and other various aromatic compounds. Flavonoids are well-known plant compounds which have antioxidant, antibacterial, antifungal, antiviral and anti-inflammatory properties. [1]

Propolis is composed of $50 \%$ resin and vegetable balsam, $30 \%$ wax, $10 \%$ essential and aromatic oils, $5 \%$ pollen and 5\% other various substances, including organic debris. Ansorge has shown the ability of Propolis to stimulate the production of transforming growth factor (TGF) Beta 1 which is important for the differentiation of odontoblasts. It also induces the synthesis of collagen by dental pulp cells. Scheler et.al showed regeneration of the pulp by applying Propolis on injured dental pulp, while calcium hydroxide caused necrosis of the pulp chamber. During the present study, there was no sign of pulp necrosis with Propolis, while necrosis was observed in about $25 \%$ of the control calcium hydroxide group. According to A Parolia the response of pulp to propolis as a pulp capping agent was comparable to MTA and Dycal. [1]

\section{Novel Endodontic Cement (NEC)}

Recently, a new endodontic cement (NEC) consisting of different calcium compounds was developed by Asgary. Clinical uses of this cement are similar to MTA. It has good handling characteristics. NEC is also able to produce hydroxyapatite. [30]

The results of an in vivo study showed that as pulp capping materials, MTA and NEC showed similar favorable results. These results were better than calcium hydroxide. [30]

NEC consists of

-calcium oxide,

-calcium phosphate,

-calcium carbonate,

-calcium silicate,

-calcium sulfate, and

-calcium chloride. [31]

According to Saeed Asgaryno inflammation was observed in MTA and NEC groups, and in $75 \%$ of each, dentinal bridge was completely formed. [32]

\section{Emdogain (EMD)}

Emdogain Gel (BioraAB,Malmo, Sweden) is a commercial product containing EMD. Amelogenin and amelin are proteins that have been suggested to participate in the final differentiation of odontoblasts and subsequent dentine formation during dentinogenesis. According to Bosshardt (2008), commercial Emdogain consist of enamel matrix derivate, water and a carrier, i.e. propylene glycol alginate.In addition, EMD contains non-amelogenin proteins including enamelin, tufelin and ameloblastin. It is generally assumed that EMD also contains other biologically active factors besides enamel proteins detected TGF-b1- or TGF-b- (transforming growth factor), like substances in EMD, and suggested that they are the main functional components of the product. In addition, EMD has been documented to contain a BMP-like growth factor (bone morphogenic protein), which belongs to the TGF-b family. According to H. Olsson Postoperative symptoms were less frequent in the EMDgel-treated teeth than in those treated with calcium hydroxide. Postoperative symptoms were less frequent in the EMDgel-treated teeth than in those treated with calcium hydroxide.EMD contains BMP like molecules and BMP expressing cells. BMP like molecules in EMD promote odontoblast differentiation and reparative dentin formation. EMD suppresses the inflammatory cytokine production by immunocytes and contains TGF-b like molecules which creates favorable environment for promoting wound healing in the injured pulp tissues.

Nakamura $Y$ et al concluded that amount of hard tissue formed in EMD treated teeth was more than twice that of the calcium hydroxide treated control teeth. Al-Hezaimi K stated MTA produced a better quality reparative hard tissue response with the adjunctive use of EMD compared with calcium hydroxide.An immunohistochemical study by Nakamuraet al (2004), demonstrated that enamel matrix proteins were present as an insoluble protein matrix in detectableamounts at the application site for about 4 weeks. These findings demonstrate that enamel matrix molecules have the capacity to induce rapid pulpal wound healing in pulpotomized teeth, and suggest that presence of enamel matrix nanospheres at the application site stimulates growth and repair of dentin. The formation of dentine islands and dentine bridge below amputation site and along dentine walls suggests that EMD treatment not only stimulates pre-existing odontoblasts, but also recruits 
new odontoblasts of unknown origin from the central part of the pulp through mimicking biological mediators normally active during early dentinogenesis. [35]

\title{
Odontogenic Ameloblast Associated Protein (Odam)
}

Odontogenic ameloblast-associatedprotein (ODAM) has been shown to be specifically expressedin ameloblasts and odontoblasts and has been suggested to play a role in the mineralization of the enamel.Odontogenic ameloblast-associated protein (ODAM) and both belong to the secretory calcium-binding phosphoprotein family, which is critical to biomineralization in vertebrates. In mammals, both ODAM is expressed by ameloblasts in the maturation stage, when immature enamel grows into a hypermineralized inorganic tissue. According to In-Seok Yangthe recombinant Odontogenic Ameloblast Associated Protein (rODAM) group showed a much lower inflammatory response than the White MTA groups. Limited reaction of odontoblasts resulted in normal pulp tissue appearance without excessive tertiary dentin formation and obliteration of the pulp cavity. rODAM accelerates reactionary dentin formation close to the pulp exposure area, thereby preserving normal odontoblasts in the remaining pulp.[36]

\author{
Endo Sequence Root Repair Material \\ It consists of \\ -Calcium silicates, \\ -monobasic calcium phosphate, \\ -zirconium oxide, \\ -tantalum oxide, \\ -proprietary fillers \\ -and thickening agents.
}

Hirschman et al., compared Cytotoxicity of MTA-Angelus, Brasseler Endosequence Root Repair Putty (ERRP), Dycal and Ultra-blend Plus (UBP)-(light curable $\mathrm{Ca}(\mathrm{OH}) 2$ ) and concluded that ERRP and UBP are less cytotoxic.[38]

\section{Castor Oil Bean (COB) Cement}

Castor oil bean cement (COB) is a new material that has been used as an endodontic sealer, and is a candidate material for direct pulp capping. According to Camargo SE after 50 days, significantly more inflammatory cells were observed in the calcium hydroxide group than in the castor oil bean group. These results demonstrate that the $\mathrm{COB}$ cement induces less inflammatory response within long periods. [39]

\section{Theracal}

It is a new light-curable pulp-capping material composed of resin and calcium silicate (Portland cement).

According to study done by M. G. Gandolfi TheraCal displayed higher calcium releasing ability and lower solubility than either ProRoot MTA or Dycal. The capability of TheraCal to be cured to a depth of 1.7 $\mathrm{mm}$ may avoid the risk of untimely dissolution. These properties offer major advantages in direct pulp-capping treatments.[40]

\section{Conclusion}

Although many products have been suggested, a recent Cochrane Review found that evidence is lacking as to the most appropriate pulp capping material. In addition, various factors are believed to influence the success of both direct and indirect pulp capping. Proper case selection, isolation, proper handling of pulp capping materials can enhance success rate.

\section{References}

[1]. A Parolia, M Kundabala, NN Rao, SR Acharya, P Agrawal, M Mohan, M Thomas, A comparative Histological Analysis Of Human Pulp Following Direct Pulp Capping With Propolis, Mineral Trioxide Aggregate And Dycal, Australian Dental Journal 2010; 55: 59-64 Thomas J Hilton, Keys To Clinical Success With Pulp Capping: A Review Of Theliterature, Oper Dent. 2009 ; 34(5): 615-625.

[3]. Cohen Bd, Paula J. Waterhouse, John M. Whitworth, Joe H. Camp, And Anna B. Fuks, 10th Edition Chapter 23 • Pediatric Endodontics, Page 809

[4]. Karthikeson P.S ,Jayalakshmi S, Pulp Capping Agents-A Review, J. Pharm. Sci. \& Res. Vol. 8(6), 2016, 525-527

[5]. Asma Qureshi1, Soujanyae., Nandakumar, Pratapkumar, Sambashivarao, Recent Advances In Pulp Capping Materials: An Overview,

[6]. Journal Of Clinical And Diagnostic Research. 2014 Jan, Vol-8(1): 316-321

[7]. Grossman's Endodontic Practice, Vital Pulp Therapy, Pulpotomy, And Apexification, $12^{\text {th }}$ Edition, 310-341

[8]. R. Kemal Su'bay, Selminasci, Human Pulpal Response To Hydroxyapatite And A Calcium Hydroxide Material As Direct Capping Agents, Oral Surgery, Oral Medicine, Oral Pathology, Volume 76, Issue 4, October 1993, Pages 485-492

[9]. Karin Cristina Da Silva Modena1, Leslie Caroll Casas-Apayco1, Maria Teresa Atta2, Carlos Alberto De Souza Costa3,

[10]. Josimeri Hebling4, Carla Renata Sipert5, Maria Fidela De Lima Navarro6, Carlos Ferreira Santos7, Cytotoxicity And Biocompatibility Of Direct And Indirect Pulp Capping Materials, Oral Surgery, Oral Medicine, Oral Pathologyvolume 76, Issue 4, October 1993, Pages 485-492

[11]. M Suzuki • A Katsumi • R Watanabe M Shirono • Y Katoh, Effects Of An Experimentally Developed Adhesive Resin System And Co2 Laser Irradiation On Direct Pulp Capping,Operative Dentistry, November/December 2005 • Volume 30 • Number 6 • 669-790 
[12]. Carlos Alberto De Souza Costa, Biocompatibility Of Resin-Based Materials Used As Pulp-Capping Agents, International Endodontic Journal Volume 36, Issue 12 December 2003 Pages 831-839

[13]. M.G. Mantellini1, T.M. Botero1, P. Yaman1, J.B. Dennison1, C.T. Hanks2, And J.E. Nör1,Adhesive Resin Induces Apoptosis And CellCycle Arrest Of Pulp Cells,J Dent Res 82(8) 2003

[14]. Karthikeson P.S Et Al/J. Pharm. Sci. \& Res. Vol. 8(6), 2016, 525-527

[15]. Chaung Hm, Hong Ch, Chiang Cp, Lin Sk, Kuo Ys, Lan Wh, Hsieh Cc, Comparison Of Calcium Phosphate Cement Mixture And Pure Calcium Hydroxide As Direct Pulp-Capping Agents. Journal Of The Formosan Medical Association = Taiwan Yizhi 1996, 95(7):545-550]

[16]. R. M. Frank,Journal Of Applied Biomaterials ,Pulp Capping With Synthetic Hydroxyapatite In Human Premolars, Volume 2, Issue 4Winter 1991 ,Pages 243-250

[17]. Takashi Komabayashi, Arataebihara, And Akira Aoki, The Use Of Lasers For Direct Pulp Capping, Journal Of Oral Science, Vol. 57, No. 4, 277-286, 2015

[18]. G. Olivi, M.D. Genovese, P. Maturo, R. Docimo,Pulp Capping: Advantages Of Using Laser Technology European Journal Of Paediatric Dentistry $\bullet 2 / 2007$

[19]. Ali Eskandarizadeh, Mohammad Hossein Shahpasandzadeh, Mahdieh Shahpasandzadeh1, Moloktorabi, Masoudparirokha Comparative Study On Dental Pulp Response To Calcium Hydroxide, White And Grey Mineral Trioxideaggregate As Pulp Capping Agents,Dental Traumatology, Volume 21, Issue 3 June 2005 Pages 150-154

[20]. M. Aeinehchi1, B.Eslami, M. Ghanbariha\& A. S. Saffar, Mineral Trioxide Aggregate (Mta) And Calcium Hydroxide As Pulp-Capping Agents In Human Teeth: A Preliminary Report, International Endodontic Journal, 36, 225^231, 2002

[21]. Niinuma A' Newly Developed Resinous Direct Pulp Capping Agent Containing Calcium Hydroxide (Mtya1-Ca), Intendod J. 1999 Nov;32(6):475-83.

[22]. Anthony J. Smith, Vitality Of The Dentin-Pulp Complex In Health And Disease: Growth Factors As Key Mediators, Journal Of Dental Education Volume 67, Number 6

[23]. Wallis E. Andelin, Shahrokhshabahang, Kenneth Wright, Mahmoud Torabinejad, Identification Of Hard Tissue After Experimental Pulp Capping Using Dentin Sialoprotein (Dsp) As A Marker, Journal Of Endodonticsvolume 29, Issue 10, October 2003, Pages 646-650

[24]. Lovschall $\mathrm{H}^{1}$, Fejerskov O, Flyvbjerg A. Pulp-Capping With Recombinant Human Insulin-Like Growth Factor I (Rhigf-I) In Rat Molars.Adv Dent Res. 2001 Aug;15:108-12.

[25]. Jin Zhang, Qisheng Tu, And Jake Chen,Applications Of Transgenics In Studies Of Bone Sialoprotein,J Cell Physiol. 2009 July ; 220(1): 30 34

[26]. M. Goldberg, N. Six, F. Decupl, D, Buchl, E. Soheilimajd, J. J Lasfargues, E. Salih, L. Stanislawski, Bone SialoproteinInduced Reparative Dentinogenesis In The Pulp Of Rat's Molar, Clin Oral Investig. 2000 Jun;4(2):110-9.

[27]. Markus K,Christoph H D, Edgar S, Till D; Shear Bond Strength Of Biodentine, Proroot Mta, Glass Ionomer Cement And Composite Resin On Human Dentine Ex Vivo; Head \& Face Medicine 2015; 11:14;1-8. 8

[28]. Alicjanowicka, Mariusz Lipski, Miroslaw Parafiniuk, Katarzynasporniak-Tutak, Damian Lichota, Anita Kosierkiewicz, Wojciechkaczmarek, And Jadwiga Buczkowska-Radli_Nska, Response Of Human Dental Pulp Capped With Biodentine And neral Trioxide Aggregate, Joe - Volume 39, Number 6, June 2013

[29]. Sun-Ju Kim, Kyung-San Min, Hyun-Wook Ryu, Hwa-Jeong Lee, Eun-Cheol Kim, The Role Of Heme Oxygenase-1 In The Proliferation And Odontoblastic Differentiation Of Human Dental Pulp Cells ,Journal Of Endodonticsvolume 36, Issue 8, August 2010, Pages 1326-1331

[30]. Kyung-San Min, Young-Man Lee, Sung-Ok Hong, Eun- Cheol Kim, Simvastatin Promotes Odontoblastic Differentiation And Expression Of Angiogenic Factors Via Heme Oxygenase-1 In Primary Cultured Human Dental Pulp Cells, Journal Of Endodontics, Volume 36, Issue 3, March 2010, Pages 447-452

[31]. Zohrehahangari, Et Al,Effect Of Propolis On Dentin Regeneration And The Potential Role Of Dental Pulp Stem Cell In Guinea Pigs,Cell Journal(Yakhteh), Vol 13, No 4, Winter 2012

[32]. Jamileh Ghoddusi1* Dds, Ms, Jaliltavakkol Afshari2 Phd, Zakiyeh Donyavi3 Dds, Azam Brook4, Reza Disfani Dds, Ms5, And Mohammad Esmaeelzadeh6 Dds

[33]. Zarrabi Mh, Javidi M, Jafarian Ah, Joushan B. Histologic Assessment Of Human Pulp Response To Capping With Mineral Trioxide Aggregate And Novel Endodontic Cement. J Endod. 2010; 36(11): 1778-81.

[34]. Saeed Asgary, Mohammad Jafareghbal, Masoudparirokh, Farzinghanavati, Hessamrahimi, A Comparative Study Of Histologic Response To Different Pulp Capping Materials And A Novel Endodontic Cement ,Oral Surgery, Oral Medicine, Oral Pathology, Oral Radiology, And Endodontologyvolume 106, Issue 4, October 2008, Pages 609-614

[35]. H. Olsson, J. R. Davies, K. E. Holst, U. Schro“ Der \& K. Petersson, Dental Pulp Capping: Effect Of Emdogain Gel On Experimentally Exposed Human Pulps, International Endodontic Journal, 38, 186-194, 2005

[36]. Bosshardt Dd. Biological Mediators And Periodontal Regeneration: A Review Of Enamel Matrix Proteins At The Cellular And Molecular Levels. J Clinperiodontol 2008;35:87-105.

[37]. Tarun Ahuja, Anuraaggurtu, Anurag Singhal, Ankita Mehrotra, Sonali Roy,The Use Of Emdogain In Endodontic Procedures,Journal Of Dental Sciences And Oral Rehabilitation, October-December 2014;5(4):205-208

[38]. In-Seok Yang, Dong-Seol Lee, Jong-Tae Park, Heung-Joong Kim, Ho-Hyun Son, And Joo-Cheol Park, Tertiary Dentin Formation After Direct Pulp Capping With Odontogenic Ameloblast-Associated Protein In Rat Teeth, Joe - Volume 36, Number 12, December 2010

[39]. Kazuhiko Kawasaki' Odontogenic Ameloblast-Associated Protein (Odam) And Amelotin: Major Players In Hypermineralization Of Enamel And Enameloid,Journal Of Oral Biosciences, Volume 55, Issue 2, May 2013, Pages 85-90

[40]. Hirschman Wr, Wheater Ma, Bringas Js, Hoen Mm. Cytotoxicity Comparison Of Three Current Direct Pulp-Capping Agents With A New Bioceramic Root Repair Putty. J Endod. 2012; 38(3): 385-8.

[41]. Camargo Se, Rode Sde M, Do Prado Rf, Carvalho Yr, Camargo Ch, Subcutaneous Tissue Reaction To Castor Oil Bean And Calcium Hydroxide In Rats, J Appl Oral Sci. 2010 May-Jun;18(3):273-8.

[42]. Gandolfi Mg, Siboni F, Prati C. ,Chemical-Physical Properties Of Theracal, A Novel Light-Curable Mta-Like Material For Pulp Cappingintendod J. 2012 Jun;45(6):571-9 